\title{
SQUAMOUS CELL CARCINOMA METASTASIS OF THE MAMMARY GLAND: CASE REPORT
}

\author{
Marcelo Moreno¹, Amauri de Oliveira¹, Tália Cássia Boff¹, Gabriela Nogueira Matschinski¹, Izadora Czarnobai \\ 'Universidade Federal da Fronteira Sul - Chapecó (SC), Brazil.
}

Introduction: Primary squamous cell carcinoma (SCC) of the breast is a rare neoplasm, which represents less than $0.1 \%$ of invasive breast cancers. Therefore, it is essential to discriminate between a primary SCC and a metastatic SCC. In order to be considered a primary carcinoma of the breast, a histological examination of the lesion must show more than $90 \%$ of squamous neoplastic cells, in addition to the absence of cutaneous SCC or other anatomical sites. Extra-mammary neoplasm metastases are uncommon, representing $0.5 \%$ to $2 \%$ of breast malignancies. Metastatic SCC in the mammary gland is an uncommon event. To date, only three cases were reported in the literature of secondary involvement of vulvar SCC in the mammary gland. The objective of this work is to report the case of a patient with secondary mammary metastasis to a vulva SCC. Case report: A 74-year-old female patient who underwent radical modified vulvectomy 10 years before. Her pathological stage was characterized as IIIB. For this reason, she was also submitted to adjuvant treatment with chemotherapy associated with radiotherapy to the vulvar region, inguinal lymph node chains and pelvic arteries. On the ninth year of cancer follow-up, she presented recurrence in the vaginal wall. In the complementary image exams, an extentension of neoplasia to pelvic organs was identified, but no distant metastatic lesions were found. She underwent monobloc resection of pelvic organs, with reconstruction of the urinary and intestinal transits. The patient showed a good clinical evolution, with no pelvic complaints. After one year, the patient returned complaining of a nodule in the right breast. On physical examination, a lesion was observed at the junction of the lateral quadrants of the breast, measuring $+/-3.5 \mathrm{~cm}$, with associated inflammatory signs and imprecise limits, with a central region showing a fistulous orifice through which the necrotic material passed. On the mammography, a dense, rounded and partially delimited lesion was identified. She underwent a core biopsy that described a SCC. According to her clinical history, it was considered a remote relapse of the vulvar SCC. The patient was submitted to a quadrantectomy with an ipsilateral axillary lymphadenectomy and reconstruction with a lateral thoracic flap. On an anatomopathological examination there was a description that the neoplasm would invade the underlying muscle tissue; and the resection margins were free. Four out of the fourteen isolated axillary lymph nodes had metastases, without perinodal soft tissue invasion. Six months after breast surgery, the patient evolved metastases to both lungs and soon after she died without response to the systemic treatment employed. This report was approved by the Research Ethics - UFFS (Universidade Federal da Fronteira Sul) (number 4.034.565). 